\title{
SURJECTIVITY OF THE PERIOD MAP IN THE CASE OF QUARTIC SURFACES AND SEXTIC DOUBLE PLANES
}

\author{
BY JAYANT SHAH
}

Communicated by Hyman Bass, April 23, 1976

Let $D$ be the period space of algebraic $K 3$ surfaces, the generic point of which corresponds to either a sextic double plane or a quartic surface [1], [2]

THEOREM 1. To every point of $D$, there corresponds a unique $K 3$ surface.

The uniqueness has been proved by Piatetskiī-Šapiro and Šafarević. The proof of existence is outlined below.

Let $o$ be the closed point of $A=\operatorname{Spec} \mathbf{C}[[t]]$. Let $A_{n}$ be the finite covering of $A$ obtained by extracting an $n$th root of $t$; let $o_{n}$ be the closed point of $A_{n}$. A family of surfaces over $A_{n}$ is a flat, projective map $f: X_{n} \rightarrow A_{n}$ such that the generic fiber is smooth, connected, and two dimensional. The special fiber over $o_{n}$ will be denoted by $\bar{X}_{n}$. A family of surfaces is said to have ordinary singularities if $\bar{X}_{n}$ is reduced and has nonsingular components crossing normally. A modification of a family $f: X \rightarrow A$ is a family $f_{n}: X_{n} \rightarrow A_{n}$ together with an isomorphism of the generic fiber of $f_{n}$ with the pull-back of the generic fiber of $f$. Recall that a family of sextic double planes or quartic surfaces, $f: X \rightarrow A$, induces a map of the generic point of $A$ into $D$; the map extends to a map $\pi: A \rightarrow D$ if and only if the monodromy group is finite [2]. The existence part of Theorem 1 follows from

Theorem 1'.1 Given any family $f: X \rightarrow A$ of sextic double planes such that the monodromy group is finite, there exists a modification $f_{n}: X_{n} \rightarrow A_{n}$ for some $n$ such that the special fiber $\bar{X}_{n}$ is reduced, irreducible and has at most rational double points as its singularities.

Theorem 1". Given any family $f: X \rightarrow A$ of quartic surfaces such that the monodromy group is finite, there exists a modification $f_{n}: X_{n} \rightarrow A_{n}$ for some $n$, with ordinary singularities, such that a component of $\bar{X}_{n}$ is a K3 surface whose periods correspond to the point $\pi(o)$ in $D$.

It follows from Mumford's Geometric Invariant Theory [4] that in order

AMS (MOS) subject classifications (1970). Primary 14D05, 14D20, 14J10, 14J15, $14 \mathrm{~J} 25$.

Key words and phrases. K3 surfaces, monodromy of singular surfaces.

${ }^{1}$ This theorem has also been proved by E. Horikawa [3]. 
to prove Theorems $1^{\prime}$ and $1^{\prime \prime}$, it is enough to analyze only those families $f: X \rightarrow A$ such that the special fiber $\bar{X}$ is semistable and belongs to a minimal orbit. We say that $\bar{X}$ has degenerate singularities if one of the following types of surfaces lie in the closure of the orbit of $\bar{X}$ :

(i) a sextic double plane which is ramified over a nonsingular quadric curve with multiplicity three;

(ii) a quartic with a cuspidal nodal curve $C$ where $C$ is either (a) a nonsingular conic or (b) a twisted cubic curve;

(iii) a quartic consisting of two nonsingular quadrics intersecting in a nonsingular conic with multiplicity two;

(iv) a nonsingular quadric with multiplicity two.

If $\bar{X}$ does not have a degenerate singularity, its singularities are called quasiordinary. A family of surfaces over $A_{n}$ is said to have quasi-ordinary singularities if its special fiber has only quasi-ordinary singularities.

Families with quasi-ordinary singularities. For any surface with quasiordinary singularities, we define its dual complex, $(K, I)$, consisting of a $\mathrm{CW}$ complex $K$ with coefficients $I$. For a reduced surface with nonsingular components crossing normally, its CW-complex is the same as Mumford's compact convex polyhedron associated with a toroidal embedding [5]. The associated coefficients in that case are: 0 for every two-dimensional cell, $H^{\mathbf{1}}(C, \mathbf{Q})$ for an edge corresponding to an intersection curve $C, H^{3}(Z, \mathbf{Q})$ for a vertex corresponding to a component $Z$.

THEOREM 2. Let $f: X \rightarrow A$ be a family of surfaces with quasi-ordinary singularities. Then, for some $n$, there exists a commutative diagram

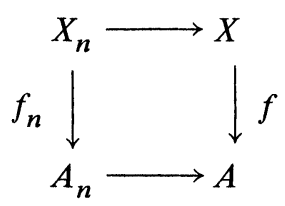

such that (i) $f_{n}: X_{n} \rightarrow A_{n}$ is a modification with ordinary singularities, and (ii) the dual complex of $\bar{X}$ and the dual complex of $\bar{X}_{n}$ are homotopic.

THEOREM 3. Let $f: X \rightarrow A$ be a family of surfaces with ordinary singularities. Let $(K, I)$ be the dual complex of $\bar{X}$. Let $2 q=$ the first Betti number of the geometric generic fiber $X_{\bar{\eta}}, \chi=$ the Euler characteristic of $K$,

$$
\begin{aligned}
x^{\prime}= & \operatorname{dim}\left\{\bigoplus_{C \text { an intersection curve in } \bar{X}} H^{1}(C, \mathrm{Q})\right\} \\
& -\operatorname{dim}\left\{\bigoplus_{Z \text { a component of } \bar{X}} H^{3}(Z, \mathbf{Q})\right\} .
\end{aligned}
$$

Then $2 q+\chi+\chi^{\prime}-1 \geqslant 0$, and the equality holds if and only if the monodromy group acting on $H^{2}\left(X_{\bar{\eta}}, \mathbf{Q}\right)$ is finite. 
Theorem 2 is proved by repeatedly blowing-up the given family; Theorem 3 is proved by calculating the spectral sequence of the Clemens' fibration [6] .

Families with degenerate singularities. The case of degenerate singularities is harder to analyze because two different families specializing to the same fiber may have monodromy groups, one of which is finite, the other infinite. However, in each of the degenerate cases listed above, the stabilizer group of the special fiber acts on the given family and, hence, on the singularities of the total space $X$. This allows us to define the geometric stability ${ }^{2}$ of the singularities of the total space and simplify these singularities using the Geometric Invariant Theory. The case is then reduced to the previous case-by blowing-up repeatedly until a family with quasi-ordinary singularities is obtained.

\section{REFERENCES}

1. I. I. Pjateckiī-Šapiro and I. R. Šafarevič, $A$ Torelli theorem for algebraic surfaces of type K3, Izv. Akad. Nauk SSSR Ser. Mat. 35 (1971), 530-572 = Math. USSR Izv. 5 (1971), 547-588. MR 44 \#1666.

2. P. A. Griffiths, Periods of integrals on algebraic manifolds: Summary of main results and discussion of open problems, Bull. Amer. Math. Soc. 76 (1970), 228-296. MR 41 \#3470.

3. E. Horikawa, Surjectivity of the period map of $K 3$ surfaces of degree 2 (to appear).

4. D. Mumford, Geometric invariant theory, Academic Press, New York; SpringerVerlag, Berlin, 1965. MR 35 \#5451.

5. G. Kempf, F. Knudsen, D. Mumford and B. Saint-Donat, Toroidal embeddings. I, Lecture Notes in Math, vol. 339, Springer-Verlag, Berlin and New York, 1973. MR 49 \#299.

6. C. H. Clemens, Jr., Picard-Lefschetz theorem for families of nonsingular algebraic varieties acquiring ordinary singularities, Trans. Amer. Math. Soc. 136 (1969), 93-108. MR 38 \#2135.

DEPARTMENT OF MATHEMATICS, NORTHEASTERN UNIVERSITY, BOSTON, MASSACHUSETTS 02115

${ }^{2}$ Horikawa's proof of Theorem 1 was helpful to me in working out this idea. My earlier proof was based upon blowing-up alone. 